\title{
Cooling reduction due to a rapid density decay of hot carriers in GaAs
}

\author{
P C M Christianen $\dagger$, E J A de Bekker $†$, H J A Bluyssent, P R Hageman $\dagger$ \\ and $M$ R Leys:
}

$\uparrow$ High Field Magnet Laboratory and Research Institute for Materials, University of Nijmegen, Toernooiveld, 6525 ED Nijmegen, The Netherlands

Eindhoven University of Technology, PO Box 513, 5600 MB Eindhoven,

The Netherlands

Abstract. The combined effect of a short carrier lifetime and of phonon emission on the cooling rate of nearly resonantly excited carriers in bulk GaAs has been investigated using picosecond time-resolved photoluminescence. Intense photoexcitation of carriers results in very short carrier density decay times of about $20 \mathrm{ps}$, leading to a considerable reduction of the cooling rate. The rapid density decay is attributed to a high stimulated recombination rate due to a high density accompanied by a low effective carrier temperature. Inclusion of the stimulated recombination in a model calculation for the energy relaxation by phonon emission leads to a significant recombination heating, which nicely explajns the observed reduction of the carrier cooling rate.

\section{Introduction}

The influence of a finite carrier lifetime is generally neglected in the theoretical analysis of picosecond cooling curves of hot electron-hole plasmas [1]. However, when the carrier lifetime becomes comparable to the time during which the photoexcited carriers relax to the band extrema through phonon emission, the energy relaxation rate due to recombination can no longer be neglected. For degenerate Fermi-Dirac carrier distributions recombination may lead to carrier heating (recombination heating) [2], which has been observed experimentally in bulk GaAs $[3,4]$. In this paper we will show, using a picosecond time- and energy-resolved photoluminescence (PL) technique, that under intense and nearly resonant excitation the carrier lifetime in GaAs is of the order of a few tens of picoseconds due to stimulated emission. resulting in a strongly reduced carrier cooling rate due to recombination heating.

\section{Experimental details and results}

The time-resolved PL experiments were carried out on two different samples, one grown by MBE, the other by MOVPE, each consisting of a $0.25 \mu \mathrm{m}$ thick undoped GaAs layer sandwiched between two ( $\mathrm{Ga}, \mathrm{Al}) \mathrm{As}$ layers [5]. Optical excitation at $1.69 \mathrm{eV}$ was achieved with picosecond light pulses from a mode-locked Ti:sapphire laser, which were focused onto the sample surface to a $20 \mu \mathrm{m}$ diameter spot. The laser time-averaged power ranged from $I_{0} / 200$ to $I_{0}\left(I_{0}=1.5 \mathrm{~kW} \mathrm{~cm}^{-2}\right)$. The picosecond
PL spectra were recorded using the up-conversion technique. The sample was kept at a temperature of $10 \mathrm{~K}$ [5].

Figure 1 shows typical measured PL spectra for two excitation densities (dotted curves). The full curves correspond to fits to the spectra calculated by the expression for band-to-band recombination, neglecting $k$-selection [6]: $r_{\text {spop }}=C \int f_{\mathrm{e}} g_{\mathrm{e}} f_{\mathrm{h}} g_{\mathrm{h}} \mathrm{d} E$, where $f_{\mathrm{e}, \mathrm{h}}\left(g_{\mathrm{e}, \mathrm{h}}\right)$ are the electron, hole Fermi-Dirac distribution (density of state) functions respectively. The coefficient $C$ contains the optical matrix element and is related to the usual radiative recombination coefficient $B_{\mathrm{r}}[6]$. From this relatively simple lineshape analysis we obtain values for

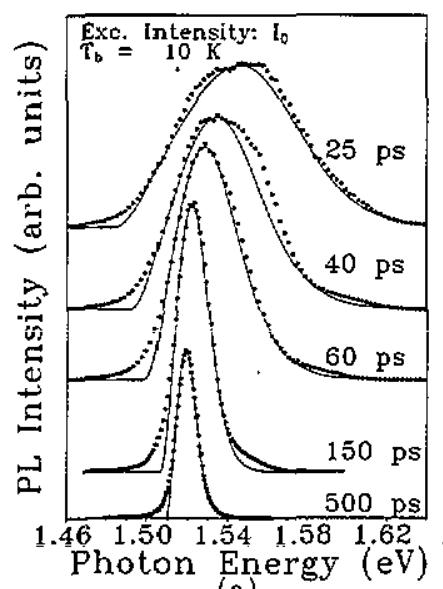

(a)

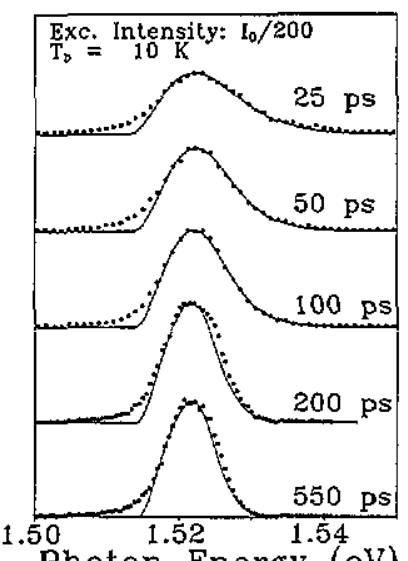

(b)
Figure 1. Measured (dotted curves) and calculated (full curves) PL spectra at different times after excitation for excitation intensities $I_{0}(a)$ and $I_{0} / 200(b)$. 


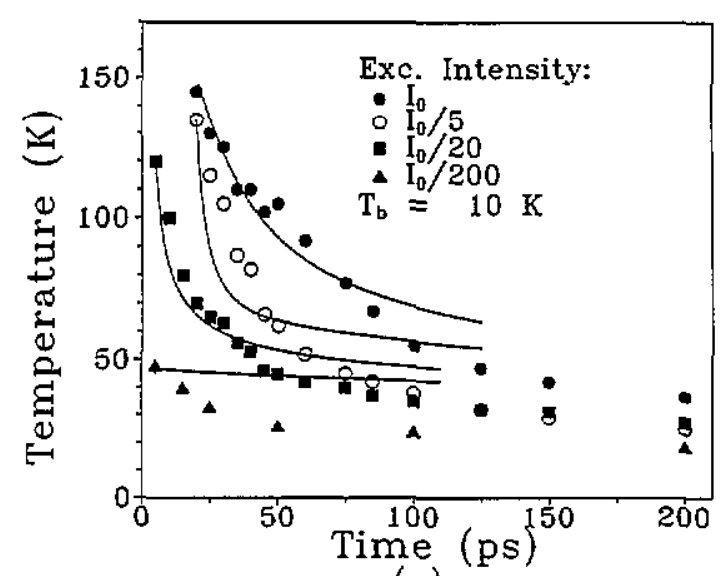

(a)

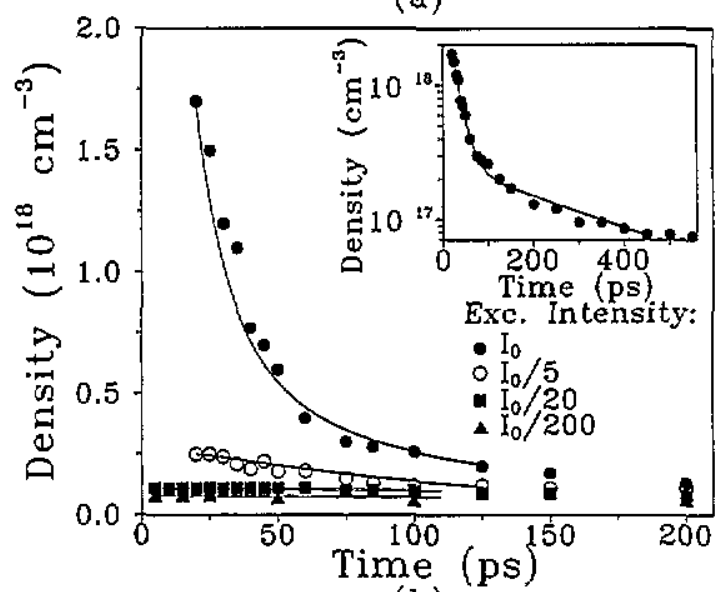

(b)

Figure 2. Carrier temperature $(a)$ and density $(b)$ as a function of time after excitation (symbols). The full curves correspond to a model calculation described in the text. The inset in $(b)$ shows the measured carrier density decay on a semilogarithmic scale (excitation level $l_{0}$ ), yielding time constants for the double exponential fit of $20 \pm 5 \mathrm{ps}$ and $200 \pm 50$ ps (full curve).

the carrier effective temperature $T_{\text {eff }}$ and density $n_{\mathrm{e}, \mathrm{h}}$, which are equal to the values deduced by a more elaborate analysis [5], including $k$-selection and collision broadening of the single-carrier states [7].

Figure 2 shows the time evolutions of $T_{\mathrm{eff}}(a)$ and $n_{\mathrm{e}, \mathrm{h}}$ (b) for different excitation intensities. The full curves were calculated from an energy relaxation model which will be discussed later. For the highest excitation intensity $\left(I_{0}\right)$ a rapid decrease of the carrier density (time constant $\sim 20 \mathrm{ps}$, full curve in the inset of figure $2(b)$ ) is observed, which forms a striking contrast to the more gradual density decay for the three lowest excitation levels. The rapid carrier density decay is attributed to a high stimulated recombination rate, which is initiated by spontaneous luminescence travelling through the photoexcited region [8]: $r_{\text {stim }} \sim r_{\text {spon }} \mathrm{e}^{g l}$ in which $l$ is the travelling distance of the luminescence and $g$ is the gain [6]. The total recombination rate $r_{\text {tot }}$ is then given by the integral of $r_{\text {stim }}$ over the photoexcited disc, assumed to be homogeneously excited with a volume equal to the laser spot diameter times the thickness of the GaAs epilayer. The high stimulated recombination rate in this experiment is a direct result of a low $T_{\text {eff }}$ together with a high $n_{\mathrm{e}, \mathrm{h}}$ after excitation. Since the electrons are created with an excess energy below the satellite valleys in the conduction band, the heating effect of electrons returning to the central valley [9] is negligible, leading to a relatively low $T_{\text {eff }}$. In a similar experiment in which electrons were created with similar densities, but much higher in the band, higher temperatures were found and no rapid density decay was observed [10].

\section{Discussion}

In order to calculate the full curves of figure 2 the radiative recombination rate, including stimulated recombination, is inserted into a model for the carrier cooling rates due to phonon emission [11]. We start from initial (experimental) values for $T_{\text {eff }}$ and $n_{\mathrm{e}, \mathrm{h}}$ from which the average carrier energy $\langle E\rangle$ is determined. After a time interval $\Delta t$ a new density $n^{\prime}$ is calculated from the total recombination rate

$$
n_{\mathrm{e}, \mathrm{h}}^{\prime}=n_{\mathrm{e}, \mathrm{h}}-\Delta t\left(\int_{0}^{\infty} \mathrm{d} E r_{\mathrm{tot}}(E)\right)
$$

Subsequently the new average carrier energy $\left\langle E^{\prime}\right\rangle$ is calculated

$$
\left\langle E^{\prime}\right\rangle=\frac{1}{n_{\mathrm{e}, \mathrm{h}}^{\prime}}\left\{\langle E\rangle n_{c, h}-\Delta t\left[\left(\frac{\mathrm{d} \mathscr{E}}{\mathrm{d} t}\right)_{\mathrm{c}-\mathrm{ph}}+\left(\frac{\mathrm{d} \mathscr{E}}{\mathrm{d} t}\right)_{\mathrm{rec}}\right]\right\}
$$

where $(\mathrm{d} \mathscr{E} / \mathrm{d} t)_{\text {rec }}$ is the energy relaxation rate due to recombination of the carriers and $(\mathrm{d} \mathscr{E} / \mathrm{d} t)_{\mathrm{c}-\mathrm{ph}}$ is the energy relaxation rate due to carrier-phonon interactions [11], including the polar optical (Lo), non-polar optical deformation, piezoacoustic and acoustic deformation interactions of both electrons and holes. In front of the electron relaxation rate due to Lo phonon scattering a phenomenological parameter $\alpha(\leqslant 1)$ [3] is used, to account for phonon heating $[7,10]$.

From $n^{\prime}$ and $\left\langle E^{\prime}\right\rangle$ a new value for the effective carrier temperature at $t=\Delta t$ is calculated. For the subsequent time intervals this procedure is repeated. All curves of figure 2 were calculated with $\Delta t=100 \mathrm{fs}, \alpha=0.3$ and a recombination coefficient $B_{\mathrm{r}}=7 \times 10^{-9} \mathrm{~cm}^{3} \mathrm{~s}^{-1}$ (at $77 \mathrm{~K})$.

The computed curves for $n_{\mathrm{c}, \mathrm{b}}(t)$ agree very well with the experimental data for all values of the excitation intensity. However, for $T_{\text {eff }}(t)$ good agreement is only obtained for $T_{\text {eff }}>60 \mathrm{~K}$ and $20<t<60 \mathrm{ps}$, the regime where stimulated emission plays a dominant role. The rather high value of $\alpha(0.3)$ suggests that phonon heating does not particularly affect the cooling at times $>20 \mathrm{ps}$ after nearly resonant excitation. For $t>60 \mathrm{ps}$ the calculated curves tend towards a final temperature of $\sim 40 \mathrm{~K}$ which is the threshold for to phonon emission. However, to describe the experimental $T_{\text {eff }}$, which decreases below $40 \mathrm{~K}$ for $t>60 \mathrm{ps}$, we have to incorporate other scattering mechanisms into our model [5].

The influence of the very short carrier lifetime on the carrier cooling rate is clearly visible in figure $2(a)$ by comparison of $T_{\text {eff }}(t)$ for excitation intensities $I_{0}$ and $I_{0} / 5$. 


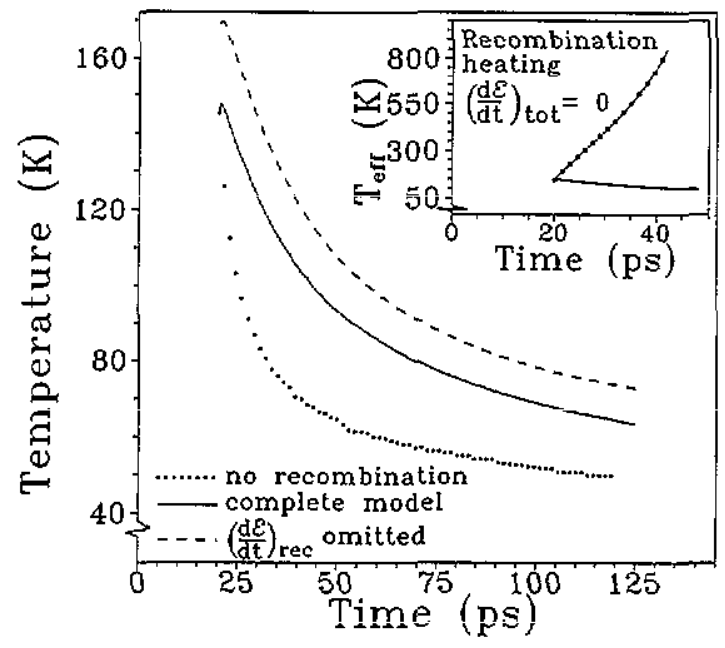

Figure 3. Time evolution of $\tau_{\text {eff }}$ calculated using the complete model (full curves), in which case recombination is neglected (dotted curves), or in the case (d $\mathscr{E} / \mathrm{d} t)_{\text {rec }}=0$ (broken curves). The inset shows the hypothetical case in which no energy relaxation is present (full circles) in comparison with the complete model calculation (full curve). All calculations start from the initial experimental values for the excitation level $I_{0}$.

At $t=20 \mathrm{ps}, \Delta T_{\text {eff }}=T_{\text {eff }}\left(I_{0}\right)-T_{\text {eff }}\left(I_{0} / 5\right) \sim 10 \mathrm{~K}$, in $=$ creases to $\Delta T_{\text {eff }} \sim 40 \mathrm{~K}$ at $60 \mathrm{ps}$, and diminishes subsequently. Thus a reduced cooling rate appears for an excitation level $I_{0}$ with respect to $I_{0} / 5$, just at those times where a considerable density decay rate is present, and which is well described by the model calculation. Hence, the observed reduction of the cooling rate can be attributed completely to the fast density decay rate, without further phonon heating effects.

Figure 3 illustrates the bare effect of a short carrier lifetime on the carrier cooling, and the relative importance of the contributing mechanisms. The full curve corresponds to the full curves in figure 2 for $I_{0}$ excitation. The broken curve corresponds to calculations in which $(\mathrm{d} \mathscr{E} / \mathrm{d} t)_{\mathrm{rec}}$ was omitted. The dotted curve was calculated by neglecting any recombination. Obviously in the presence of a rapidly decaying. density (full curve) the cooling rate is reduced considerably with respect to the case of no density decay (dotted curve). This reduction is the consequence of two effects:

(i) The rapid carrier density decay heats the carrier distribution, which is known as recombination heating $[2,3]$. This effect originates from the first term on the right-hand side of equation (2): $\left\langle E^{\prime}\right\rangle=\left(n / n^{\prime}\right)\langle E\rangle$, and is simulated by the chain curve in the inset of figure 3 , calculated by omitting $(\mathrm{d} \mathscr{E} / \mathrm{d} t)_{\text {tot }}$ for the experi= mentally observed carrier density decay for excitation level $I_{0}$. For our experimental parameters we deduce an enormous heating from $145 \mathrm{~K}$ to $800 \mathrm{~K}$ within $40 \mathrm{ps}$. These effects are considerably larger than the recombination heating discussed previously $[2,3]$, because of our exceptionally short carrier lifetimes of about $20 \mathrm{ps}$. However, due to the large Lo phonon cooling rates no real heating is observed experimentally, but merely a reduction in the cooling rate.

(ii) Due to the rapid carrier recombination rate the loss of energy of the recombining carriers $(\mathrm{d} \mathscr{E} / \mathrm{d} t)_{\mathrm{rec}}$ forms an extra energy relaxation channel. Neglecting $(\mathrm{d} \mathscr{E} / \mathrm{d} t)_{\text {rec }}$ in the calculations leads to the broken curves in figure 3. Comparison with the full curve proves that $(\mathrm{d} \mathscr{E} / \mathrm{d} t)_{\text {rec }}$ enhances the cooling conspicuously and thus definitely cannot be neglected.

\section{Conclusion}

Nearly resonant excitation of hot carriers in $\mathrm{GaAs}$ results in an extraordinarily fast density decay which can be well explained by stimulated recombination. Consequently the radiative recombination rate has to be included in the analysis of the energy relaxation rate, since the short carrier lifetime leads to a considerable reduction of the carrier cooling by means of recombination heating, opposed partially by the extra loss of energy of the recombining carriers, which enhances the cooling rate.

\section{Acknowledgments}

Part of this work was supported by the 'Stichting voor Fundamenteel Onderzoek der Materie' (FOM) with financial supportit of the 'Nederlandse Organisatie voor Wetenschappelijk Onderzoek' (NWO).

\section{References}

[1] Shah J 1986 IEEE J. Quantum Electron. 221728

[2] Bimberg D and Mycielski J 1986 J. Phys. C: Solid State Phys. 192363

[3] Leo K and Rühle W W 1987 Solid State Commun. 62659

[4] Christianen P C M, Reinen H A I M, Bluyssen H I A and Leys M R 1990 Proc. 20th Int. Conf. on the Physics of Semiconductors ed E M Anastassakis and J D Joannopoulos (Singapore: World Scientific) p 2526

[5] Christianen P C M 1993 PhD Thesis University of Nijmegen

Christianen P C M, de Bekker E J A, Reinen H A $J$ M, Bluyssen $H J$ A, Leys $M R$ and Hageman $\mathbf{P} \mathbf{R}$ to be published

[6] Lasher G and Stern F 1964 Phys. Rev. 133 A553

[7] Collet $\mathrm{J} H$, Rühle W W, Pugnet $M$, Leo $\mathrm{K}$ and Million A 1989 Phys. Rev. B 4012296

[8] Dubard J, Oudar J L, Alexandre F, Hulin D and Orszag A 1987 Appl. Phys. Lett. 50821

[9] Shah J, Deveaud B, Damen T C, Tsang W T, Gossard A C and Lugli P 1987 Phys. Rev. Lett. 592222

[10] Hollering R W J, Berendschot T T J M, Bluyssen H J A Reinen H A J M, Wyder P and Roozenboom F 1988 Phys. Rev. B 3813323

[11] Pugnet M, Collet J and Cornet A 1981 Solid State Commun. 38531 